\title{
Acta de constitución de la Red de Laboratorios Públicos de Medicamentos para producción, investigación, desarrollo y servicios
}

En la Ciudad Autónoma de Buenos Aires, a los 13 días del mes de septiembre de 2007, a las 17:00 hs., se reúnen en la calle Paraguay $N^{\circ} 21551^{\circ}$, las siguientes personas:

Dr. Jorge RACHID, de Laboratorios Puntanos (San Luis), Dra. Cecilia MILAZZO, de la Unidad de Producción de Medicamentos (FCE-UNLP), Lic. Pascual FIDELIO y Dr. Jorge ZARZUR, del Laboratorio de Hemoderivados "Pte. A. U. Illia" (U.N.C.), Isabel Haydee CASADEI, Laboratorio LAFORMED S.A. (Formosa), Farm. Elbio Antonio SAIDMAN, del Laboratorio de Control de Calidad de Medicamentos U.N.S.L. (San Luis), Lic. Carlos DEBANDI, de la Agencia Córdoba Ciencia S. E. (Córdoba), Dra. Viviana DABBENE, del Laboratorio de Análisis Farmacéutico de la Unidad CEPROCOR de la Agencia Córdoba Ciencia S.E., Cdor. Luis Alberto CAMPO y Dr. Héctor J. LUSI, del Laboratorio de Medicamentos Genéricos Pampeano de la Municipalidad de Gral. Pico (La Pampa), Dra. Martha GATTUSO y Dr. Nelson STURTZ, de Facultad de Ciencias Bioquímicas y Farmacéuticas (UNR), Sr. Juan Carlos COMUZZI, del Laboratorio Elmetec de la UTN, Dr. Horacio BARRI, del Laboratorio Farmacia Municipal de Córdoba, Ricardo SAAD, del Laboratorio Productor de Medicamentos de Río Negro (PROZOME), Bqco. Sergio A. MAROEVICH, de Laboratorio de Especialidades Medicinales S.E. de la Municipalidad de Rosario, Farm. Mercedes L. STEIN del Laboratorio de Especialidades Medicinales del Ministerio de Salud Pública de Misiones (LEMis), Dr. Pedro HOLCMAN, de Laboratorio de Hurlingam, Ing. Orlando COSTA, del Laboratorio de Medicamentos del Hospital San Luis de la Municipalidad de Bragado, Dra. Alicia BARDÓN, del Centro de Elaboración y Estudios Farmacéuticos de la Facultad de Bioquímica, Química y Farmacia de la Universidad Nacional de Tucumán, Farm. Ana María RONCO, del Laboratorio de la Municipalidad de Olavaria, Farm. Gladys M. ORELLANO y Farm. Nancy E. CIVERCHIA, del Laboratorio Productor de Medicamentos de Mendoza (Lapromed), Dr. Gustavo RíOS, de ANLIS, Farm. Héctor A. GUGLIELMONE, de la Planta de Medicamentos de Corrientes del Ministerio de Salud Pública de la Provincia de Corrientes (PLA.ME.COR), con el fin de dar conformación a una RED DE LABORATORIOS PÚBLICOS DE MEDICAMENTOS PARA PRODUCCIÓN, INVESTIGACIÓN, DESARROLLO Y SERVICIOS que tendrá por objeto unir voluntades para llevar a cabo articulaciones entre los convocantes a los fines de estimular la producción pública de medicamentos de acuerdo a las mejores pautas de calidad e implementando buenas prácticas de fabricación, así como propender la investigación y el desarrollo de nuevos productos, formulaciones, aplicaciones, métodos y todo aquello relacionado al campo de los medicamentos y su implicancia con la mejora de la salud de la población, con la correspondiente relevancia y pertinencia social para el país. Todas las acciones que se desarrollen dentro de la RED serán adoptadas de común acuerdo o por acuerdo de partes y se llevarán a cabo utilizando los instrumentos que ellas consideren más apropiados.

Para el logro de tales objetivos, la RED DE LABORATORIOS PÚBLICOS DE MEDICAMENTOS PARA PRODUCCIÓN, INVESTIGACIÓN, DESARROLLO Y SERVICIOS podrá realizar las siguientes actividades: 
A) Reunirse con otras organizaciones sociales tanto locales, regionales como nacionales e internacionales, además de oficinas gubernamentales, con el propósito de celebrar acciones de cooperación técnica y de formación de recursos humanos, acciones de difusión, adquisición en conjunto de insumos o equipamiento, intercambio (y/o venta) de productos y servicios, gestión conjunta de financiamiento, cumplimiento de normas de calidad (BPM), así como toda acción que se considere relevantes para propósitos fundacionales de la RED.

B) Organización de eventos, actividades y proyectos que abarquen áreas de trabajo de la RED, la investigación y el desarrollo de proyectos específicos.

C) Realizar talleres, cursos y encuentros de capacitación en el área formal y no formal sobre temas relacionados a la problemática de los medicamentos y de la salud poblacional.

La enunciación precedente no tiene carácter limitativo, por lo que la RED podrá realizar actividades necesarias y/o convenientes para el mejor cumplimiento de su objeto y en ningún caso importarán directa o indirectamente beneficios y/o ventajas económicas para sus asociados en forma personal.

Luego de deliberar los asistentes deciden formalizar la creación de dicha RED a la que se le dará el nombre de "RELAP".

En la reunión son asignadas las siguientes personas: Dr. Jorge Rachid, Dra. Cecilia Milazzo y Lic. Pascual Fidelio, como representantes de "RELAP" ante las entidades públicas y privadas cuando éstas convoquen a temas inherentes a los objetivos básicos que generaron la presente sociedad.

Asimismo se fija como domicilio de referencia de la RED en la Ciudad Autónoma de Buenos Aires en Cerrito $5124^{\circ}$ of. 5 y Av. Viamonte 1719, (1055), $9^{\circ}$ piso, of. 40 /41.

El conjunto de los firmantes se compromete a participar en la elaboración de un reglamento de funcionamiento de la RED a fin de explicitar los mecanismos operativos de funcionamiento, así como a solicitar ante las autoridades de sus respectivas instituciones la acreditación que formalice su representatividad ante la RED. 